\title{
Primary Care Access and Emergency Room Use Among Older Veterans
}

\author{
Rachel M. Werner, MD, $P h D^{1,2,3}$, Anna Canamucio, $M S^{7}$, Steven C. Marcus, $P h D^{1,3,4}$, and \\ Christian Terwiesch, $P h D^{7,3,5}$
}

${ }^{1}$ Center for Evaluation of Patient-Aligned Care Teams, Philadelphia VAMC, Philadelphia, PA, USA; ${ }^{2}$ Division of General Internal Medicine, Perelman School of Medicine at the University of Pennsylvania, Philadelphia, PA, USA; ${ }^{3}$ Leonard Davis Institute of Health Economics, University of Pennsylvania, Philadelphia, PA, USA; ${ }^{4}$ School of Social Policy and Practice, University of Pennsylvania, Philadelphia, PA, USA; ${ }^{5}$ eepartment of Operations and Information Management, the Wharton School, University of Pennsylvania, Philadelphia, PA, USA.

BACKGROUND: Patient access to primary care is often noted to be poor. Improving access may reduce emergency room (ER) visits.

OBJECTIVE: To examine the relationship between primary care access and ER use and to test whether this relationship is moderated by having a continuous relationship with a Primary Care Provider (PCP) (or if the PCP is the near-sole provider of care for patients).

DESIGN AND PATIENTS: A longitudinal retrospective study of 627,276 patients receiving primary care from 6,398 primary care providers (PCPs) nationally within the Veterans Health Administration (VHA) in 2009. We tracked weekly changes in PCP-level appointment availability.

MEASUREMENTS: The number of a PCP's patients who went to the ER in a given week.

RESULTS: Among all PCPs, being absent from patient care for the week had no effect on whether that PCP's patients used the ER in that week (incident rate ratio (IRR) 0.997, $\mathrm{p}=0.70$ ). However, among PCPs who were near-sole providers of care, a PCP's absence for a week or more had a statistically significant effect on ER visits (IRR 1.04, p=0.01). The percentage of a PCP's weekly appointment slots that were fully booked (booking density) had no significant effect on whether their patients used the ER in that week among all PCPs. However, among near-sole providers of care, a 10percentage point increase in the booking density changed the IRR of ER visits in that week by 1.005 $(\mathrm{p}=0.08)$ and by 1.006 on weekdays $(\mathrm{p}=0.07)$.

CONCLUSIONS: Patients' access to their PCP had a small effect on whether those patients used the ER among PCPs whose patients rarely saw another PCP. Among other PCPs, there was no effect of PCP access on ER use. These results suggest that sharing patient-care responsibilities across PCPs may be effective in improving access to care and decreasing unnecessary ER use.

KEY WORDS: access to care; primary care; continuity of care; veterans.

J Gen Intern Med 29(Suppl 2):S689-94

DOI: $10.1007 / \mathrm{s} 11606-013-2678-8$

(C) Society of General Internal Medicine 2013

Published online April 9, 2014
Patient access to primary care providers (PCPs) is often noted to be poor. ${ }^{1-3}$ A 1999 Kaiser Family Foundation survey reported that $27 \%$ of adults reported difficulty gaining timely access to a health care provider. ${ }^{2}$ Between 1997 and 2001, the percentage of people reporting difficulty obtaining timely care rose from $23 \%$ to $33 \%{ }^{4}$ In a 2001 survey, $43 \%$ of adults reported being unable to get timely care for an urgent health condition. ${ }^{5}$ More recently, a 2006 survey reported that only $27 \%$ of adults with a usual source of care could easily contact their physician or obtain timely care. $^{6}$

Delays in timely access to care have been associated with decreased patient satisfaction, ${ }^{7}$ and have prompted concerns that such delays may lead to inappropriately high rates of emergency room (ER) visits. Indeed, ER visits for nonurgent conditions are common, accounting for up to $40 \%$ of all ER visits. ${ }^{8}$ Improving timely primary care access has thus become a major focus in primary care for the past decade, and recently many providers have restructured their practices to improve patient access to care. ${ }^{9,10}$

However, evidence linking poor primary care access to ER overuse is mixed. Several surveys of ER patients reported a link between self-reported primary care access and ER use. ${ }^{11}$ For example, a 2006 survey of ER patients reported poor primary care access as one of the main reasons for ER use. ${ }^{12}$ Another study found that ER use is higher among patients who self-report worse access to primary care. ${ }^{13}$ Other research examined whether ER use is higher among patients with worse primary care access using non-self-reported measures of primary care access. ${ }^{14-19}$ These studies generally focus on the correlations between ER use and primary care characteristics such as lack of a regular physician or poor continuity of care, and find that ER use rises as continuity and having a usual source of care falls. However, without direct measures of primary care access (i.e. how easy it is to get an appointment when needed $)^{15-19}$ and by relying on cross-sectional relationships, these studies may mismeasure the relationship between practice characteristics and ER use. 
To get more directly at the relationship between primary care access and ER use, a few studies used changes in scheduling procedures designed to increase timely primary care access (i.e. implementation of open-access scheduling) to study the relationship between primary care access and ER visits. However, to our knowledge, the studies using this approach examined changes at a single site or in a small group of clinics, limiting generalizability. While one study found a decrease in urgent care visits with the implementation of open-access scheduling, ${ }^{20}$ neither it or another study found a consistent effect of open access on ER visits. $^{20,21}$ Thus, the relationship between primary care access and ER use remains questionable.

The effect of primary care access on ER use may depend in part on whether a patient has a continuous relationship with his or her PCP (also called interpersonal continuity). ${ }^{22}$ On one hand, having an ongoing and continuous relationship with a PCP has been found to be associated with improved patient satisfaction ${ }^{23}$ and clinical care outcomes. ${ }^{24}$ This effect on improved care may extend to fewer ER visits. On the other hand, PCPs who provide more continuous care may be less accessible in the case of an emergency, ${ }^{25,26}$ and may thus increase ER use. The evidence on this question is scant, but the tension between continuity and improving access in primary care is particularly relevant as primary care moves toward team-based care models such as the medical home.

Our objective in this study is two-fold. Our primary aim is to examine the relationship between primary care access and ER use. Our secondary aim is to test whether this relationship is moderated by having a continuous relationship with a PCP. We use longitudinal data to examine how changes in a provider's availability affect ER use by that provider's patients. We measure provider availability using primary care scheduling data, enabling us to estimate weekly urgent care (or unscheduled) capacity in over 6,000 PCPs nationally. We do this among a large, national population of US patients in the Veterans Health Administration (VHA).

\section{DESIGN AND MEASUREMENT}

\section{Data}

We used two data sources in this study. First, we used data from the VHA's Corporate Data Warehouse, a national repository of VHA data from several clinical and administrative systems that contain data on all patient encounters within the VHA and include consistent provider and patient identifying information. We used this data to identify all primary care and ER visits within the VHA. Second, we used VA-Medicare data from the VA Information Resource Center, which contained all Medicare claims for our cohort who were dually enrolled in the VHA and Medicare to identify ER use outside of the VHA.

\section{Study Population}

Using these data sources, we identified all veterans receiving primary care within the VHA in 2009 and their primary care practitioners (PCPs). We identified a cohort of primary care enrollees as all patients with at least one primary care visit in 2008 and alive on January 1, 2009 ( $n=$ $4,181,611$ patients). This ensured that all patients included in the 2009 cohort had an established relationship with a PCP at the VHA. We then assigned these patients to PCPs who were practicing in the VHA in 2009 (i.e. had scheduled primary care appointments in 2009; $n=4,067,418$ patients). We used a standard attribution rule to assign patients to PCPs, assigning patients to the PCP they saw most frequently that year. In cases of ties (where a patient saw two PCPs the same number of times), we assigned the patient to the tied PCP seen first in 2009. We included only patients assigned to a PCP with a panel of between 75 and 2,000 patients, as extreme outliers in panel size were likely due to data errors $(n=3,983,922$ patients assigned to 6,426 PCPs). This was the cohort we use to measure one of our independent variables, PCP booking density (described below).

To measure ER use (our dependent variable), we further refined our study sample. We included only patients who were 65 years or older, and thus eligible for Medicare $(n=$ $1,920,253$ patients assigned to 6,414 PCPs) and those patients who did not have Medicare claims for primary care visits in 2008 or 2009, and thus exclusively used VHA for primary care $(n=627,276$ patients assigned to 6,398 PCPs). We limited the sample to those eligible for Medicare because we could observe all ER visits for this group, whether they occurred within or outside of the VHA. We limited the sample to those receiving all primary care from the VHA, because we could accurately observe PCP access for this group. For those receiving primary care outside of the VHA, we could not observe how accessible their nonVHA PCP was. Since access to a non-VHA PCP could affect ER use within the VHA, if we did include veterans receiving primary care outside of VHA, this unobserved factor could have biased our results. Although this limited the number of patients in our sample, it did not substantially change the number of PCPs in the sample, which are the unit of observation for this study.

\section{Independent Variables: PCP Availability}

We measured PCP availability in two ways. First, we measured whether the PCP was absent from seeing patients in a given week by creating a weekly dichotomous measure 
indicating whether the assigned PCP was not seeing patients (i.e. had zero patient appointments for the entire week), as would be the case for vacations or other leaves of absence.

Second, we created a continuous measure of availability during the weeks the PCP was seeing patients - the PCP's booking density. Booking density was defined as the number of a PCP's appointment slots that were booked in a week divided by the PCP's total number of appointment slots that week. Conceptually, this represents the percentage of a PCP's time that was unavailable if a patient requested an urgent appointment that week, where higher values suggest less available time to accommodate urgent visits. We measured available appointments as a percentage of all appointments (rather than the absolute number of appointments) to normalize across clinical sites, as some sites routinely use shorter appointments, thus scheduling more appointments in a week. For part-time providers who do not see patients every weekday, we counted non-patient weekdays as fully booked (booking density of $100 \%$ ) and averaged the daily booking densities for the week. For all providers, we accounted for week-long absences from patient care (e.g. vacations) by considering those weeks as fully booked.

\section{Dependent Variable: ER Use}

Our main dependent variable was a provider-level weekly count of the number of a PCP's patients who went to the ER in a given week. Our measure of ER use included ER visits within the VHA and outside of the VHA (i.e. covered by Medicare). Although $16 \%$ of VHA sites reported no ER visits during our study period, these sites tended to be small ( $8 \%$ of PCPs), mostly outpatient facilities, and exclusion of these sites did not substantively alter the results.

\section{Analysis}

In our main analysis, we used a negative binomial model to estimate the weekly effect of PCP availability on their patients' ER use overall, on weekends (when availability should have less of an effect), on weekdays (when the effect of availability should be concentrated), and for ER visits that were treatable in primary care (which should be most sensitive to PCP availability). We defined primary care treatable ER visit based on the New York University algorithm. ${ }^{27}$ We used this algorithm to classify ER visits as non-emergent, emergent but treatable in primary care, or emergent with ER care needed. We classified the first two groups (non-emergent and emergent but treatable in primary care) as primary care treatable ER visits.

In all models, we included medical center-level booking density as a covariate, week fixed effects (to control for seasonal variation), and PCP fixed effects. PCP fixed effects both controlled for unobserved time-invariant PCP character- istics that might affect the relationship between booking density and patient ER use, and allowed each PCP to serve as a control for him or herself. Thus, we estimated within-PCP effects of changes in booking density over the course of the year on patient ER use. This takes advantage of the longitudinal nature of our data and provides estimates of the effect that are less prone to bias than typical estimates from cross-sectional data.

We ran all analyses in two groups of PCPs. First, we included all PCPs described above in the study population. Second, because the relationship between primary care access and ER use may be moderated in part by whether or not a PCP tends to be the sole provider of care, we stratified our sample by those PCPs who did and did not provide the vast majority of care to their patients, or interpersonal continuity. ${ }^{22}$ To estimate interpersonal continuity, we calculated the proportion of a patient's visits with his or her assigned PCP. By taking the proportion of non-assigned visits averaged across all patients in a PCP's panel, we got a measure of each PCP's style of care with respect to providing continuous care - that is, how often a PCP's panel saw his or her own PCP rather than a substitute PCP. For PCPs whose panel of patients saw them at least $95 \%$ of the time ( $\mathrm{n}=2,527$ PCPs or $39 \%$ of all PCPs in our cohort), we defined them as providing continuous care to their patients. All analyses adjusted standard errors for clustering of observations within PCP.

\section{RESULTS}

Patients were predominantly male and on average 75 years old (Table 1). The average patient visited the ER less than

Table 1. Characteristics of Study Sample

\begin{tabular}{|c|c|}
\hline \multicolumn{2}{|l|}{ Patient characteristics $(n=627,276)$} \\
\hline Age, mean (SD) & $74.6(7.0)$ \\
\hline Male, $\%$ & 98.2 \\
\hline White race, $\%$ & 85.8 \\
\hline Black race, $\%$ & 10.8 \\
\hline $\begin{array}{l}\text { Number of ER visits per patient in } 2009 \text {, } \\
\text { mean (SD) }\end{array}$ & $0.4(1.1)$ \\
\hline \multicolumn{2}{|l|}{ PCP characteristics $(n=6,398)$} \\
\hline $\mathrm{MD}$ or $\mathrm{DO}, \%$ & 72.2 \\
\hline NP or PA, $\%$ & 27.8 \\
\hline PCP's practice located in a medical center, $\%$ & 51.1 \\
\hline PCP's practice located in rural area, $\%$ & 21.2 \\
\hline $\begin{array}{l}\text { Number of patients in a PCP panel, mean } \\
\text { (SD) }\end{array}$ & $619.9(334.3)$ \\
\hline $\begin{array}{l}\text { For assigned panel, } \% \text { of visits with assigned } \\
\text { PCP, mean (SD) }\end{array}$ & $89.1(10.9)$ \\
\hline $\begin{array}{l}\text { Number of weeks absent from outpatient care, } \\
\text { mean (SD) }\end{array}$ & $7.0(10.0)$ \\
\hline Booking density, mean (SD) & $82.5(15.4)$ \\
\hline \multicolumn{2}{|l|}{ Practice characteristics $(n=850)$} \\
\hline Number of PCPs in a practice, mean (SD) & $7.6(8.9)$ \\
\hline Number of patients in a practice, mean (SD) & $4682(5099)$ \\
\hline \multicolumn{2}{|l|}{ ER visits per $\mathrm{PCP} /$ week } \\
\hline Total ER visits per week, mean (SD) & $0.7(1.2)$ \\
\hline $\begin{array}{l}\text { Primary care treatable ER visits per week, } \\
\text { mean (SD) }\end{array}$ & $0.3(0.7)$ \\
\hline
\end{tabular}


Table 2. The Effect of a PCP Being Absent from Patient Care on the Rate of Other Types of Patient Visits Among that PCP's Patients

\begin{tabular}{|c|c|c|c|c|c|c|}
\hline & \multicolumn{3}{|c|}{ Among all PCPs } & \multicolumn{3}{|c|}{$\begin{array}{l}\text { Among PCPs whose patients rarely see non- } \\
\text { assigned PCPs }\end{array}$} \\
\hline & \multicolumn{3}{|c|}{$(n=6,398)$} & \multicolumn{3}{|c|}{$(n=2,527)$} \\
\hline & IRR & $95 \%$ CI & $P$ value & IRR & $95 \% \mathrm{CI}$ & $P$ value \\
\hline Total ER visits & 0.997 & $(0.980$ to 1.014$)$ & 0.70 & 1.036 & (1.008 to 1.066$)$ & 0.01 \\
\hline Weekday ER visits & 1.001 & $(0.982$ to 1.020$)$ & 0.96 & 1.040 & (1.008 to 1.074$)$ & 0.01 \\
\hline Weekend ER visits & 0.983 & $(0.950$ to 1.017$)$ & 0.33 & 1.026 & $(0.967$ to 1.089$)$ & 0.39 \\
\hline Primary care treatable ER visits & 1.010 & $(0.985$ to 1.035$)$ & 0.45 & 1.080 & (1.036 to 1.124$)$ & 0.00 \\
\hline
\end{tabular}

once in 2009 ( 0.4 times per year). The majority of their PCPs were physicians, over half of whom practiced in a medical center (i.e. a hospital-based clinic). On average, there were seven PCPs and over 4,600 patients in each practice. The average PCPs was $83 \%$ booked, and his or her panel of patients saw their assigned PCP $89 \%$ of the time.

Among all PCPs, being absent from patient care for the week had no effect on whether that PCP's patients used the ER in that week (Table 2). The incident rate ratio (IRR) of ER visits among patients' whose PCP was absent for the week was $0.997(p=0.70)$. There was also no statistically significant relationship between PCP absence and ER use on weekdays, weekends, or for primary care treatable ER visits.

However, among PCPs whose patients saw them at least $95 \%$ of the time, the effect of being absent for the week on ER visits was statistically significant (Table 2). In weeks when a PCP was absent, the IRR for an ER visits was 1.04 $(p=0.01)$, or an increase in the incidence of ER visits of $4 \%$. This effect was consistent for weekday ER visits (IRR 1.04; $\mathrm{p}=0.01$ ), but was not statistically different from zero for weekend ER visits, when PCP availability should have little effect on ER use (IRR 1.026; $p=0.39$ ). The effect of PCP absence was also statistically significant for primary care treatable ER visits (IRR 1.08; $\mathrm{p}<0.001$ ).

Among all PCPs, booking density had no significant effect on whether their patients used the ER in that week (Table 3). A 10-percentage point increase in booking density changed the IRR of ER visits in that week by $1.002(p=0.15)$. The effect of booking density remained close to zero and non-statistically significant for weekday ER visits and weekend ER visits (IRR $1.003[\mathrm{p}=0.13]$ and $0.999[\mathrm{p}=0.80]$, respectively) and for primary care treatable ER visits (IRR 1.003; $\mathrm{p}=0.19$ ).

Among PCPs whose patients saw them at least $95 \%$ of the time, the effect of PCP booking density was, in some cases, statistically significant (Table 3). A 10-percentage point increase in the booking density was associated with a marginally statistically significant positive IRR of ER visits in that week by $1.005(p=0.08)$ overall, by $1.006(p=0.07)$ for weekday ER visits, and by $1.010(\mathrm{p}=0.02)$ for primary care treatable ER visits. There was no effect of booking density on weekend ER visits, as expected.

\section{CONCLUSION}

We found that patient access to their PCP (as measured by PCP absence from clinical care) was statistically significantly associated with incidence of ER use, but only in settings where patients rarely saw a substitute PCP. Also, a PCP's booking density affected whether his or her patients used the ER among patients who rarely saw another PCP, though this effect was small. Among PCPs whose patients did not routinely receive continuous care from that PCP (e.g. saw other, non-assigned PCPs for at least $5 \%$ of visits), there was no effect of PCP access on ER use for either absence from patient care or for booking density.

Table 3. The Effect of a 10-Percentage Point Increase in PCP Booking Density on the Rate of ER Visits Among that PCP's Patients

\begin{tabular}{|c|c|c|c|c|c|c|}
\hline & \multicolumn{3}{|c|}{ Among all PCPs } & \multicolumn{3}{|c|}{$\begin{array}{l}\text { Among PCPs whose patients rarely see non- } \\
\text { assigned PCPs }\end{array}$} \\
\hline & \multicolumn{3}{|c|}{$(n=6,398)$} & \multicolumn{3}{|c|}{$(n=2,527)$} \\
\hline & IRR & $95 \%$ CI & $P$ value & IRR & $95 \%$ CI & $P$ value \\
\hline Total ER visits & 1.002 & (0.999 to 1.006$)$ & 0.15 & 1.005 & $(0.999$ to 1.010$)$ & 0.08 \\
\hline Weekday ER visits & 1.003 & (0.999 to 1.007$)$ & 0.13 & 1.006 & (1.000 to 1.012$)$ & 0.07 \\
\hline Weekend ER visits & 0.999 & (0.993 to 1.006$)$ & 0.80 & 1.002 & $(0.991$ to 1.013$)$ & 0.72 \\
\hline Primary care treatable ER visits & 1.003 & (0.998 to 1.008$)$ & 0.19 & 1.010 & (1.002 to 1.018$)$ & 0.02 \\
\hline
\end{tabular}


The effect of PCP availability on ER use was modest. However, an increase in ER visits of $4 \%$ translates into a meaningful number of ER visits. Based on our study cohort, there were over 97,000 ER visits in 2009 among patients whose PCPs were the near-sole provider of care. Our study implies that by implementing systems that promote shared responsibility for patients over continuity of care and ensuring sufficient capacity to accommodate patient access to primary care, the VA could eliminate close to 4,000 ER visits annually.

These results also highlight the tension between access to care and continuity of care. Continuity of care has long been seen as a central tenet of primary care. ${ }^{28}$ Indeed, past work has found that higher continuity is associated with higher patient satisfaction ${ }^{23}$ and, in some studies, better clinical outcomes. $^{24,26}$ However, pressures to improve access to primary care, including use of same-day visits and open-access scheduling, threaten to reduce interpersonal continuity, as improving PCP availability often means reducing the ability to manage patients as a sole provider. Indeed, some prior work has shown that efforts to improve access have resulted in a decline in continuity. ${ }^{25,29-31}$ Our results support this finding. Among PCPs who were the sole providers for their patients, PCP absence was associated with increased ER use.

Our results highlight several important lessons. First, they are consistent with prior research suggesting that simple interventions like advanced-access scheduling may not solve the problem of ER overuse. ${ }^{20,21}$ Our results suggest that the challenge of improving primary care access is more complex than simply increasing appointment availability, and may only work in certain settings where patients rely on a single PCP for their care. As the VHA implements the medical home model nationwide, it will be important to remember that open access alone may not effectively reduce ER use.

Additionally, our results offer some insight into ways to potentially improve primary care access. PCP availability had an effect on ER use only among PCPs who appeared to be sole providers of care of their patients, but not PCPs who shared the care of their patients with colleagues. This suggests that providing a team-based approach rather than interpersonal continuity of care may be an effective way to improve access to care and decrease unnecessary ER use. PCPs with higher interpersonal continuity of care were not simply located in rural locations or practices with few other PCPs (results not reported), suggesting that team-based practice styles were possible.

When interpreting our results, it is important to remember our measure of access, as well as the clinical setting of our research. Our measures of PCP availability capture information about the accessibility of established PCPs, as all patients in our sample had an ongoing relationship with a PCP. In the absence of an established PCP relationship, access may simply be impossible for some patients, who may thus face the choice between no care and care in the ER.

The clinical setting of our research is also important. Results show that within the VHA system, patients needing urgent care are generally able to obtain access, even when their PCPs are unavailable. Since the mid-1990s, the VHA has invested heavily in the establishment and growth of primary care. More recently, the VHA has begun investing in team-based approaches to primary care, such as the medical home model. It is possible that the VHA system has created sufficient operational flexibility such that patients seeking urgent care have an alternative to using the ER, even when faced with a high booking density of their PCP. This may negate the effect of PCP availability on ER use in our study.

Our measures of access and the clinical setting of the VHA limit the generalizability of our results. In addition, the criteria we used to create our study cohort, including limiting it to those patients over the age of 65 and who do not get primary care outside of the VHA, further limit the generalizability of our results. However, these exclusions are important to improve the internal validity of our study, as this is the cohort for whom we can most accurately observe use of primary care and ER. Despite these limitations, to our knowledge, our research provides the largest study to date examining the effect of PCP availability on ER use, providing new and robust evidence about the relationship between primary care availability and the rate of ER use.

Acknowledgements: This work was undertaken as part of the Veterans Administration's PACT Demonstration Laboratory initiative, supporting and evaluating VA's transition to a patient-centered medical home. Funding for the PACT Demonstration Laboratory initiative is provided by the VA Office of Patient Care Services. The views expressed in this article are those of the authors and do not necessarily reflect the position or policy of the Department of Veterans Affairs or other affiliated institutions.

Conflict of Interest: The authors declare that they do not have a conflict of interest.

Corresponding Author: Rachel M. Werner, MD, PhD; Blockley Hall, room 1204, 423 Guardian Drive, Philadelphia, PA 19104, USA (e-mail: rwerner@upenn.edu).

\section{REFERENCES}

1. Merritt HA. Summary report: 2009 survey of physician appointment wait times. 2009. http://www.merritthawkins.com/pdf/ mha2009waittimesurvey.pdf. Accessed September 5, 2013.

2. Kaiser Family Foundation. National survey of consumer experiences with health plans. Menlo Park: The Henry J. Kaiser Family Foundation; 2000 . 
3. Trude S, Ginsburg PB. Issue Brief: An update on Medicare beneficiary access to physician services: Center for Studying Health System Change 2005.

4. Strunk BC, Cunningham PJ. Treading water: American's access to needed medical care, 1997-2001. Washington: Center for Studying Health System Change; 2002.

5. Greenblatt J. Statistical brief \#8: Access to urgent medical care, 2001. Rockville: Agency for Healthcare Research and Quality; 2002.

6. Beal AC, Doty M, Hernandez S, Shea $\mathbf{K}$, Davis $\mathbf{K}$. Closing the divide: how medical homes promote equity in health care. New York: Commonwealth Fund; 2007.

7. Murray M, Bodenheimer T, Rittenhouse D, Grumbach K. Improving timely access to primary care. JAMA. 2003;289(8):1042-6.

8. Billings J, Parikh N, Mijanovich T. Issue brief: Emergency department use in New York City: substitute for primary care? New York: The Commonwealth Fund; 2000.

9. Rose KD, Ross JS, Horwitz LI. Advanced access scheduling outcomes: a systematic review. Arch Intern Med. 2011;171(13):1150-9. doi:10.1001/archinternmed.2011.168.

10. Murray M, Berwick DM. Advanced access: reducing waiting and delays in primary care. JAMA. 2003;289(8):1035-40. doi:10.1001/ jama.289.8.1035.

11. Cunningham PJ, Clancy CM, Cohen JW, Wilets M. The use of hospital emergency departments for nonurgent health problems: a national perspective. Med Care Res Rev. 1995;52(4):453-74.

12. Foundation CHC. Issue brief: Overuse of emergency departments among insured Californians. Oakland: California Health Care Foundation; 2006.

13. Rust G, Ye J, Baltrus P, Daniels E, Adesunloye B, Fryer GE. Practical barriers to timely primary care access: Impact on adult use of emergency department services. Arch Intern Med. 2008;168(15):1705-10. doi:10.1001/archinte.168.15.1705.

14. Lowe RA, Localio AR, Schwarz DF, Williams S, Tuton LW, Maroney S, et al. Association between primary care practice characteristics and emergency department use in a Medicaid managed care organization. Med Care. 2005;43(8):792-800.

15. Rosenblatt RA, Wright GE, Baldwin LM, Chan L, Clitherow P, Chen FM, et al. The effect of the doctor-patient relationship on emergency department use among the elderly. Am J Public Health. 2000;90(1):97102.

16. Burge F, Lawson B, Johnston G. Family physician continuity of care and emergency department use in end-of-life cancer care. Med Care. 2003;41(8):992-1001.
17. McCusker J, Ciampi A, Vadeboncoeur AM, Roberge D, Larouche D, Verdon $\mathbf{J}$, et al. Continuity of primary care and emergency department utilization among elderly people. Can Med Assoc J. 2007;177(11):1362-

18. McCusker J, Roberge D, Levesque JF, Ciampi A, Vadeboncoeur A, Larouche $\mathbf{D}$, et al. Emergency department visits and primary care among adults with chronic conditions. Med Care. 2010;48(11):972-80.

19. Gill JM, Mainous AG 3rd, Nsereko M. The effect of continuity of care on emergency department use. Arch Fam Med. 2000;9(4):333-8.

20. Solberg LI, Maciosek MV, Sperl-Hillen JAM, Crain AL, Engebretson KI, Asplin BR, et al. Does improved access to care affect utilization and costs for patients with chronic conditions. Am J Manage Care. 2004;10(10):717-22.

21. Subramanian U, Ackermann RT, Brizendine EJ, Saha C, Rosenman MB, Willis DR, et al. Effect of advanced access scheduling on processes and intermediate outcomes of diabetes care and utilization. J Gen Intern Med. 2009;24(3):327-33.

22. Saultz JW. Defining and measuring interpersonal continuity of care. Ann Fam Med. 2003;1(3):134-43.

23. Saultz JW, Albedaiwi W. Interpersonal continuity of care and patient satisfaction: a critical review. Ann Fam Med. 2004;2(5):445-51.

24. Saultz JW, Lochner J. Interpersonal continuity of care and care outcomes: a critical review. Ann Fam Med. 2005;3(2):159-66.

25. Phan K, Brown SR. Decreased continuity in a residency clinic: a consequence of open access scheduling. Fam Med. 2009;41(1):46-50.

26. Pandhi N, Saultz JW. Patients' perceptions of interpersonal continuity of care. J Am Board Fam Med. 2006; 19(4):390-7.

27. The Center for Health and Public Service Research. NYU ED algorithm. New York University. 2000. http://wagner.nyu.edu/faculty/billings/ nyued-download. Accessed September 5, 2013.

28. Donaldson MS, Yordy KD, Lohr KN, Vanselow NA. Primary care: America's health in a new era. Washington: National Academy of Sciences: Institute of Medicine; 1996.

29. Ahluwalia S, Offredy M. A qualitative study of the impact of the implementation of advanced access in primary healthcare on the working lives of general practice staff. BMC Fam Pract. 2005;6(1):39.

30. O'Connor ME, Matthews BS, Gao D. Effect of open access scheduling on missed appointments, immunizations, and continuity of care for infant well-child care visits. Arch Pediatr Adolesc Med. 2006;160(9):889

31. Salisbury C, Montgomery AA, Simons L, Sampson F, Edwards S, Baxter H, et al. Impact of Advanced Access on access, workload, and continuity: controlled before-and-after and simulated-patient study. $\mathrm{Br} \mathrm{J}$ Gen Pract. 2007;57(541):608. 